\title{
BMJ Open Long-term outcomes in patients with severe depression after in-hospital treatment - study protocol of the depression long-term Augsburg (DELTA) study
}

Inge Kirchberger (D , , Barbara Maleckar, ${ }^{1,2}$ Christine Meisinger, ${ }^{1}$ Jakob Linseisen, ${ }^{1,3}$ Max Schmauss, ${ }^{2}$ Jessica Baumgärtner ${ }^{2}$

To cite: Kirchberger I, Maleckar B, Meisinger C, et al. Long-term outcomes in patients with severe depression after in-hospital treatment - study protocol of the depression long-term Augsburg (DELTA) study. BMJ Open 2019;9:e032507. doi:10.1136/ bmjopen-2019-032507

- Prepublication history for this paper is available online. To view these files, please visit the journal online (http://dx.doi. org/10.1136/bmjopen-2019032507).

Received 21 June 2019

Revised 26 November 2019

Accepted 03 December 2019

Check for updates

(C) Author(s) (or their employer(s)) 2019. Re-use permitted under CC BY-NC. No commercial re-use. See rights and permissions. Published by BMJ.

For numbered affiliations see end of article.

Correspondence to Dr Inge Kirchberger; i.kirchberger@unika-t.de

\section{ABSTRACT}

Introduction Depressive disorders are very common diseases entailing a great burden on affected people. However, comprehensive information on long-term disease course in patients with severe depression is lacking so far. The objectives of the DELTA study are to examine long-term outcomes and their predicting factors, to assess clinical response of antidepressant pharmacotherapy by applying therapeutic drug monitoring, to identify predictors of therapeutic non-response, to describe the long-term healthcare utilisation and to investigate the role of biomarkers in disease course.

Methods and analysis A cohort study including all adult hospitalised cases (age range 18 to 75 years) of severe major depression who are admitted to the Bezirkskrankenhaus Augsburg is established. It is planned to include 300 patients. During the hospital stay, information is gathered through personal interview, self-administered questionnaires, cognitive tests and chart review. Furthermore, biomaterials are collected. After hospital discharge, patients are repeatedly reexamined over time $(3,6,12,24$ and 36 months) to collect information about mortality, relapse, depression severity, health-related quality of life (HRQOL), perceived stigma, cognitive functions, diet, physical activity, treatment and healthcare utilisation. Follow-up blood samples are collected to determine therapeutic drug levels. The primary study aim is to investigate long-term therapeutic response, survival, relapse, HRQOL and cognitive functions. Survival time and time to relapse or re-hospitalisation will be analysed using Cox regression models. Changes of HRQOL, depressive symptoms and cognitive functions over time will be examined using generalised linear regression models for repeated measures or mixed models. Correlates of the disease course will be modelled using suitable generalised linear, mixed, estimating equation and growth curve models.

Ethics and dissemination The study protocol was approved by the Ethics Committee of the LudwigMaximilians-Universität München (date of approval: 23 October 2017, reference number: 17-625). Study results will be presented at scientific conferences and published in peer-reviewed scientific journals.
Strengths and limitations of this study

- This study considers a variety of outcomes, which are rarely included in clinical trials (eg, cognitive functions, health-related quality of life).

- A limitation of the study is that analyses are not based on randomised treatment assignments.

Loss to follow-up may lead to biased results.

\section{INTRODUCTION}

Depressive disorders are very common diseases affecting 180 million people worldwide. ${ }^{1}$ Data from Germany revealed a lifetime prevalence of any depressive disorder (excluding depressive episodes in bipolar disorder) of $19 \%$ (women: $25 \%$, men $12 \%$ ) and a 12-months-prevalence of $12 \%$ in the general population aged 18 to 65 years. Severe depressions account for $30 \%$ of all cases. ${ }^{2}$ Persons with depressive disorders suffer from a reduction of health-related quality of life (HRQOL) $)^{3-5}$ and impaired functioning, specifically regarding interpersonal activities and work. ${ }^{6}$ In particular, the large indirect costs associated with absenteeism from work, disability and mortality make depressive disorders the most costly mental disorders in Europe. ${ }^{8}$

Several studies have identified treatment deficits und unmet healthcare needs in persons with depressive disorders. ${ }^{2910}$ A recent study using claims data from the German statutory health insurance showed that $18 \%$ of persons with a diagnosed severe major depression were not treated, and only $12 \%$ received a treatment according to the guidelines. ${ }^{11}$ In addition, since routine data from health insurance funds do not include information on in-hospital treatment/medication 
of depression, profound scientific knowledge on this issue is lacking so far. ${ }^{12}$ Comprehensive data on health services use and real-life treatment in Germany based on large samples is missing. Insufficient healthcare, however, may contribute to unfavourable disease courses. ${ }^{13}$

Current national and international guidelines on treatment of major depression have highlighted a number of future research needs. ${ }^{15}$ An important issue is the lack of longitudinal studies and knowledge on the continuation and maintenance phase of treatment. Another research need refers to the unclear relation between medication blood levels and therapeutic response and the long-term attainment of therapeutic blood levels. In clinical practice, partial response and non-response to antidepressant pharmacotherapy are common. ${ }^{16}$ Therapeutic drug monitoring can be used to optimise antidepressant pharmacotherapy and to avoid side effects by tailoring the drug dosage to the individual characteristics of a patient. ${ }^{17}$ However, the benefit of this tool in routine patient care is so far not adequately assessed. ${ }^{14}$

In addition, guidelines encourage to further investigate the role of complementary treatments in depression. Complementary and complementary medicine (CAM) is very popular in Germany. ${ }^{18}{ }^{19}$ Only a few interventions have demonstrated therapeutic effectiveness in depression, for example, St John's wort. ${ }^{20-22}$ However, it is unclear, how many patients use CAM in the years after hospital discharge, which CAM interventions they choose and which characteristics CAM users have.

In terms of outcomes of depression treatment, clinical trials primarily focus on symptom reduction as indicators of therapeutic response. Other indicators such as HRQOL and cognitive functioning are so far only scarcely included into investigations on depressive disorders. ${ }^{23}$

Furthermore, it is so far unclear how patients who will respond to depression treatment differ from patients who will not respond. A number of social, mental, lifestyle, physical and physiological characteristics may play a role. Current research has identified physical activity and diet as being related with the development and course of mental disorders ${ }^{24}$ and that physical activity and exercise support the improvement of depressive disorders. ${ }^{25}$ Detailed long-term analyses of the association of physical activity with relief of depressive symptoms and changes in HRQOL are, however, scarce. In addition, studies among older people have further suggested that there is a strong association between low muscle strength and depressive symptoms. ${ }^{26-28}$ However, studies addressing the relationship between muscular strength and clinical status among hospitalised patients with depression are missing and the prognostic importance of muscular strength for clinical outcomes of these patients has not yet been investigated. In addition, diet and nutrition seem to play a crucial role in depression, as demonstrated by epidemiological studies and current randomised controlled trials. ${ }^{29} 30$

Increasingly, novel '-omics' data (genomics, metabolomics, proteomics) are being used as a tool for a better understanding of psychiatric disorders, including the discovery of biomarkers for diagnosis, monitoring of disease course and treatment response and the development of more personalised treatment. ${ }^{3132}$ Moreover, the gut microbiota is in the focus of current research. The bidirectional communication between the gut microbiota and the brain has been shown to influence neurotransmission and the behaviour that is often associated with neuropsychiatric conditions. Modulation of the gut microbiota through probiotic and prebiotic foods and supplements may be a novel therapeutic approach also in depressive disorders. ${ }^{33} 34$ In addition, examination of gut microbiota may facilitate the identification of patient subgroups according to disease development and response to pharmacological treatment. In these new research areas prospective studies are needed. ${ }^{3435}$

\section{Objectives}

The primary study aim is to investigate long-term therapeutic response, survival, relapse, HRQOL and cognitive functions. Secondary study objectives are (1) to describe long-term medication use and to assess clinical response and side effects of antidepressant pharmacotherapy by applying therapeutic drug monitoring, (2) to identify predictors of therapeutic non-response, (3) to examine the role of physical activity, grip strength and diet in disease course and outcomes, (4) to examine the association between cognitive/perceptual impairment and disease course and outcomes, (5) to describe the longterm healthcare utilisation and to determine differences in healthcare utilisation according to diagnosis, age, gender, socioeconomic status, social network and place of residence, (6) to investigate the use of complementary therapies and (7) to investigate the role of biomarkers analysed in different biospecimens (blood, urine, stool), for example, inflammatory parameters, metabolomics and proteomics data in disease course and outcomes.

\section{METHODS AND ANALYSIS \\ Study design}

The DELTA study is a single-centre, prospective cohort study. Patients are recruited from the Bezirkskrankenhaus (BKH) Augsburg, which offers in-hospital psychiatric healthcare for the study region covering the city and district of Augsburg ( 400000 adult inhabitants). All patients receive a baseline assessment during their hospital stay after verification of inclusion criteria and obtainment of informed consent. Subsequently, patients are contacted at 3, 6, 12, 24 and 36 months after discharge and invited to take part in follow-up examinations at the BKH Augsburg. Patient recruitment has started in February 2018 and the study will be finished in 2023.

\section{Patient selection}

Inclusion criteria

The study will include all consecutive patients (age 18 to 75 years) with a primary discharge diagnosis of severe major depression (International Classification of 
Diseases, Tenth Revision (ICD-10) codes: F32.2, F32.3, F33.2, F33.3) according to the Structured Clinical Interview for DSM IV (SCID-I, categories A, B, C, D) ${ }^{36}$ criteria, with in-hospital treatment at the BKH Augsburg. Furthermore, a score $\geq 16$ at the Hamilton Depression Rating Scale $^{37}$ is required.

\section{Exclusion criteria}

Exclusion criteria are: (1) Mental and behavioural disorders due to psychoactive substance use (F10 to F19), (2) disorders of adult personality and behaviour (F60 to F60.9, F61) (3) mental retardation (F70 to F79), (4) dementia (F00 to F09), (5) insufficient knowledge of the German language, (6) comorbidities with a life expectancy $<1$ year (eg, terminal cancer).

\section{Measurements}

Measurements take place in the hospital during the initial hospital stay and 3, 6, 12, 24 and 36 months after discharge (see table 1) and are conducted by well-trained psychologists and study nurses. The patients are contacted by study nurses by telephone in order to make an appointment for a follow-up examination.

\section{Interview}

After information and written consent of the patient, a standardised face-to-face baseline interview is performed in order to collect information on sociodemographic characteristics, previous depressive episodes and concomitant disorders (eg, cardiovascular diseases, diabetes, cancer) and treatment and lifestyle factors such as smoking and alcohol consumption. The interview conducted at the follow-up visits additionally includes information on healthcare utilisation (readmissions, visits to physicians, psychotherapists, other health professionals, hospitals), use of complementary therapies, employment status, current medication and medication side effects.

\section{Medical charts}

Information on disease history, in-hospital treatment and medication are extracted from the patients' medical records after discharge.

\section{Clinical ratings}

Disease severity is assessed by the study psychologists using the Hamilton Depression Rating Scale. ${ }^{37}$ In addition, symptoms of depression are self-rated by the patients using the Beck Depression Inventory. ${ }^{39}$

\section{Self-adminstered questionnaires}

Self-adminstered questionnaires are used to collect information on HRQOL, diet, physical activity and perceived stigmatisation.

HRQOL is assessed by two different questionnaires. The WHOQOL-BREF is an instrument intended to measure quality of life according to the WHO definition that quality of life is the individuals perception of their position in life in the context of the culture and value systems in which they live and in relation to their goals, expectations, standards and concerns. It includes 24 items which cover the domains 'Physical', 'Psychological', 'Social relationships' and 'Environment'. The WHOQOL-BREF has been successfully applied in patients with depression and the German version has been validated in individuals with psychiatric disorders. ${ }^{40-43}$ The second questionnaire is the EQ-5D-5L, a generic instrument consisting of five items addressing mobility, self-care, usual activity, pain/discomfort and anxiety/depression and a visual analogue scale to assess general health. ${ }^{44}$ Psychometric properties of the German EQ-5D have been evaluated across diseases and populations. ${ }^{45-48}$ The main advantage of the EQ-5D-5L is the availability of utility scores which enable the calculation of quality-adjusted life-years and the incorporation in health-economic analyses. ${ }^{49}$

A food frequency questionnaire is used to obtain data on the habitual diet. ${ }^{50}$ This questionnaire comprises questions on the frequency and portion size of 158 food items eaten during the year preceding hospital admission. It has been used in a large epidemiological study (European Prospective Investigation into Cancer and Nutrition (EPIC) trial) and the German version has been tested for its reproducibility and validity within this trial. ${ }^{5051}$

Physical activity is measured by the International Physical Activity Questionnaire (IPAQ) 7-item short-form which is a well-validated and very common self-report instrument. ${ }^{52} 53$ The IPAQ has been successfully applied in studies on patients with major depression and bipolar disorder. ${ }^{545}$

The Internalised Stigma of Mental Illness (ISMI) scale is a 29-item questionnaire measuring self-stigma among persons with psychiatric disorders. ${ }^{56}$ Psychometric data are available from a number of studies demonstrating reliability and validity across a wide range of mental health conditions, languages and cultures. ${ }^{57}$ The German version of the ISMI scale has shown good psychometric properties with high internal consistency, good test-retest reliability and good construct validity among people with schizophrenia spectrum disorder. ${ }^{58}$

\section{Tests and examinations}

A subtest of the Wechsler Adult Intelligence Scale-IV - the digit span — is used to assess working memory. ${ }^{59}$ Participants are verbally presented a series of two numbers which shall be recalled in forward, backward and sequential order. The number of digits in a series increase until the participant fails to correctly recall the series twice. Executive functions include semantic word fluency as assessed by the Regensburger WortflüssigkeitsTest. ${ }^{60}$ The participant is asked to name as many different animals/foods as possible within $2 \mathrm{~min}$. Furthermore, a version of the Stroop-Test (Farbe-Wort-Interferenztest) is used to assess executive functions. ${ }^{61}$ The participants are requested to read names of colours, to name the colour of colour samples and to name the printed colour of the colour names. In the last condition that represents an interference condition, the name of the colour is printed in a colour not denoted by the name. The processing 
Table 1 Variables and measures

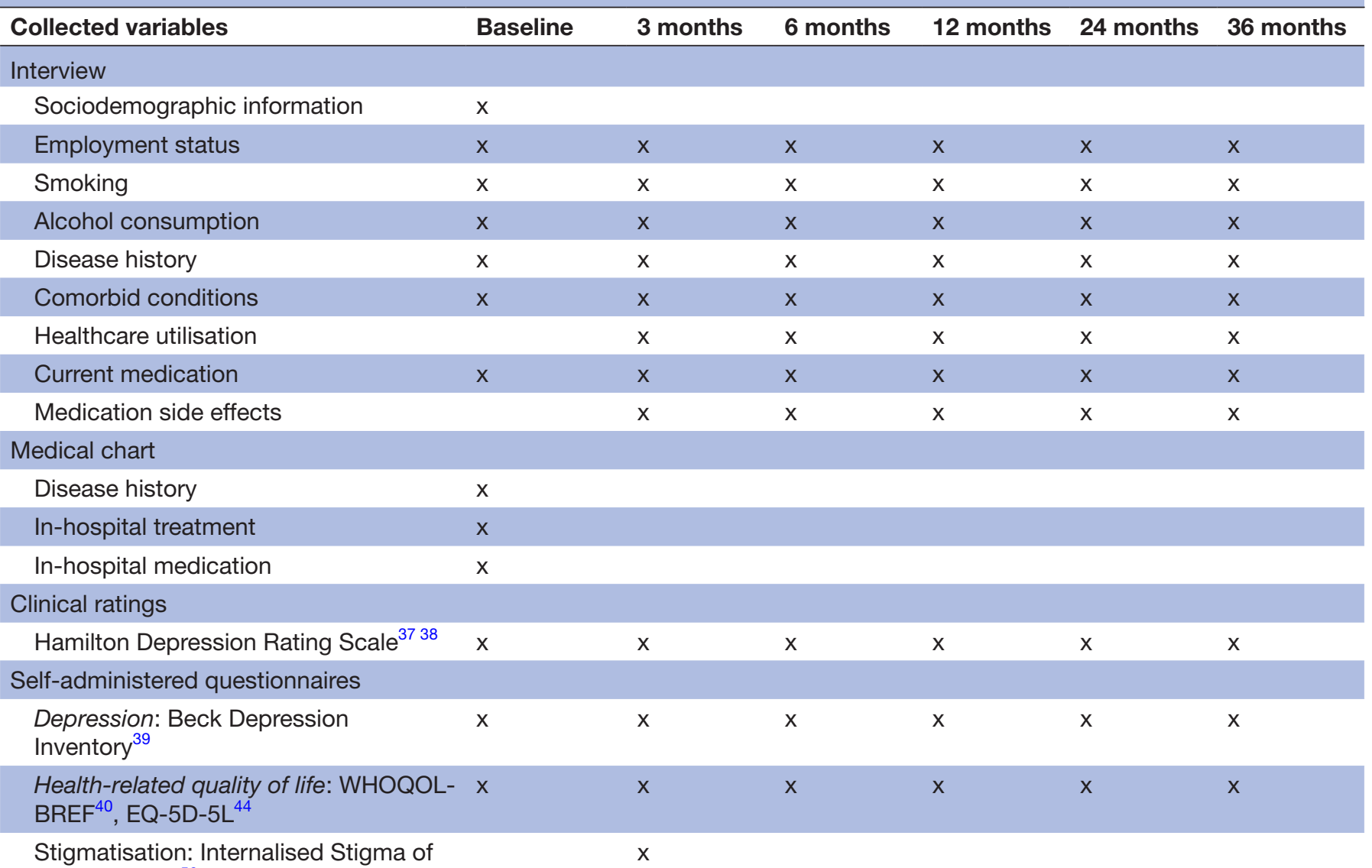
Mental Illness ${ }^{56}$

\begin{tabular}{|c|c|c|c|c|c|c|}
\hline Diet: Food Frequency Questionnaire & $x$ & & & $x$ & & \\
\hline $\begin{array}{l}\text { Physical Activity: International Physical } \\
\text { Activity Questionnaire - short-form }{ }^{52}\end{array}$ & $x$ & $\mathrm{x}$ & $x$ & $x$ & $x$ & $x$ \\
\hline $\begin{array}{l}\text { Memory: WechslerAdult Intelligence } \\
\text { Scale-IV, subtest digit span }{ }^{59}\end{array}$ & $x$ & & $\mathrm{x}$ & $\mathrm{x}$ & $\mathrm{x}$ & $x$ \\
\hline Executive functions: Farbe-Wort- & $x$ & & $x$ & $x$ & $\mathrm{x}$ & $\mathrm{x}$ \\
\hline
\end{tabular}
Interferenztest according to J.R. Stroop ${ }^{61}$

$\begin{array}{llllll}\text { Olfactory functions: Sniffin sticks-1262 } & \mathrm{x} & \mathrm{x} & \mathrm{x} & \mathrm{x} & \mathrm{x} \\ \text { Handgrip strength } & \mathrm{x} & \mathrm{x} & \mathrm{x} & \mathrm{x} & \mathrm{x} \\ \text { Body height } & \mathrm{x} & \mathrm{x} & \mathrm{x} & \mathrm{x} & \mathrm{x} \\ \text { Body weight } & \mathrm{x} & \mathrm{x} & \mathrm{x} & \mathrm{x} & \mathrm{x}\end{array}$

\section{Biomaterial}

Blood sample for therapeutic drug monitoring (small, without storage)

Blood sample (large, with storage)

Stool sample

Urine sample

$\mathrm{x}$
$\mathrm{x}$
$\mathrm{x}$
$\mathrm{x}$


time is documented as well as the number of corrected and uncorrected errors in the interference condition. It is also reported whether a repetition of a single condition was necessary.

Olfactory functions are assessed by the Sniffin' Sticks Screening 12 test, ${ }^{6263}$ a validated test for the screening of olfactory functioning. Twelve odorants are offered to the participants. The correct substance has to be chosen from a card displaying four different substances. Depending on the number of correctly recognised substances, the test is able to differentiate between anosmia, hyposmia and normosmia.

Handgrip strength is measured by means of the Jamar Plus Hydraulic Hand Dynamometer (device number: 2016011210). The measurements are performed on the grip position 2 and the test modus LR1, which means that the left and right hand are tested in turn. Prior to that, participants are questioned about their preferred hand use and whether they are aware of any restrictions for the measurements to be carried out (eg, diseases, injuries, inflammations or chirurgical operations of arms and hands). Measurements are carried out in a predetermined sitting position: Participants are instructed to grip the dynamometer as strongly as possible for $3 \mathrm{~s}$ and to breathe normally during this exertion. Each hand is tested three times in this fashion, starting with the left hand.

Body weight is measured during the baseline assessment and all of the follow-up examinations. Participants are asked to remove their shoes and any heavy jackets, belts or heavy objects from their pockets. Additionally, body height is assessed once during participants' involvement in the study.

\section{Biomaterial}

Blood, stool (native and stabilised) and urine samples are collected within the DELTA study. Blood samples are collected in the morning, fasting and before medication intake. Biomaterial samples are processed and aliquoted into sample tubes at the BKH Augsburg, frozen at $-80^{\circ} \mathrm{C}$ and stored in the biorepository of the Chair of Epidemiology, UNIKA-T Augsburg. A sample of $30 \mathrm{~mL}$ blood is collected from the patients after study inclusion and 1 year post discharge. In addition, during the hospital stay, after having reached a stability in terms of medication management and close to the date of discharge and at each follow-up visit, a second, smaller blood sample is collected and analysed for serum or plasma drug concentration. Results of the therapeutic drug monitoring are forwarded to the patients' prescribing physician, in order to enable a dose modification to reach therapeutic drug levels.

\section{Mortality follow-up}

Regular mortality follow-ups are performed using information from the residents' registration offices in the study area. Causes of death are extracted from the death certificates provided by the local health authorities in the study region and are coded by a trained person according to ICD-10.

\section{Quality assurance}

Quality assurance (QA) will be based on the 'Guidelines and Recommendations for Good Epidemiological Practice' from the German Society of Epidemiology ${ }^{64}$ and the guideline for adaptive management of data quality in cohort studies and registries published by TMF (Technologie- und Methodenplattform für die vernetzte medizinische Forschung e.V.). ${ }^{65}$

Prior to the start of the main study all applied methods and procedures were tested in five pilot patients. Standard operating procedures were established for all relevant operations. Study nurses and psychologists received a training prior to the start of the study and will receive ongoing training and yearly recertification. Particularly, study psychologists received a rater training for SCID and Hamilton Depression Rating Scale by an experienced psychiatrist.

Recruitment rates will be continuously monitored and QA of scientific data (eg, plausibility, completeness) will be regularly performed by the data managers.

\section{Statistical analysis}

Analyses of data plausibility, completeness, distribution, concordance and accuracy will be performed in order to ensure data quality. In order to estimate bias due to loss to follow-up, baseline date of complete cases and cases lost to follow-up will be compared. Descriptive statistics will be used to describe medication use and side effects, drug concentrations, healthcare utilisation, use of complementary therapies, etc. Survival time and time to relapse or re-hospitalisation will be analysed using Cox regression models. Health services utilisation, including outpatient visits after discharge from hospital and re-hospitalisations, will be examined using Poisson regression models. Changes of HRQOL, depressive symptoms and cognitive functions over time will be examined using generalised linear regression models for repeated measures or mixed models if appropriate. Correlates of the disease course will be modelled using suitable generalised linear, mixed, estimating equation and growth curve models. Covariate selection will be performed using directed acyclic graphs and related methods.

\section{Sample size estimation}

Every year, about 300 patients with severe depression receive in-hospital treatment at the BKH Augsburg. Based on the experience from previous studies, it is expected that $50 \%$ of the patients will take part in the study and $50 \%$ will be lost to follow-up. Consequently, it is planned to recruit about 300 study participants within 2 years and have available follow-up information on 150 patients. A sample size estimation for the primary study objective (to identify subgroups of treatment response) indicated that data from 126 participants are needed given an effect size of $0.25, \alpha$ of 0.05 and $\beta$ of 0.95 . 


\section{Ethics and dissemination}

The study is performed according to the Declaration of Helsinki. Written, informed consent is obtained from the study participants. Patients who do not consent will not be included. The study results will be presented at national and international conferences and published in peer-reviewed scientific journals.

\section{Patient and public involvement}

Public and patients were not involved in development of the study design, in recruitment of study participants or the implementation of the study. However, the study results will be discussed using focus groups consisting of patients and healthcare providers.

\section{DISCUSSION}

This paper describes the study design of the DELTA study, a cohort study evaluating the long-term outcomes of patients with severe depression. The DELTA study is unique in a multifold way.

First, as an observational study, it offers a real-life view in the disease course of patients with severe depression beyond the inherent restrictions of clinical trials. It is possible to investigate a variety of outcomes, which are essential from the patient perspective (eg, HRQOL, cognitive functions), but are rarely included as outcomes in clinical trials.

Moreover, results from the DELTA study may improve the understanding of non-response to antidepressive treatment, since a number of potential factors that may contribute to response or non-response are investigated. These factors include novel '-omics' data (metabolomics, proteomics) and the gut microbiota, which may play an important role for the personalisation of depression treatment in the future.

In addition, the DELTA study, which follows the participants for 3 years, is able to provide information on long-term adherence to antidepressive medication and treatment response. Comprehensive information on healthcare utilisation and treatments beyond pharmacological therapy, including CAM interventions, is collected and may elucidate strengths and weaknesses of the healthcare offered to patients with severe depression in the study region.

In summary, the DELTA study is expected to contribute to the evidence regarding the long-term disease course and healthcare utilisation in patients with severe depression. The results can be an important basis for clinicians, administrators and health policymakers to improve healthcare in patients with this disorder.

\section{Author affiliations}

${ }^{1}$ Chair of Epidemiology at UNIKA-T, Ludwig-Maximilians-Universität München, Augsburg, Germany

${ }^{2}$ Department of Psychiatry, Psychotherapy and Psychosomatics of the University Augsburg, Bezirkskrankenhaus Augsburg, Augsburg, Germany

${ }^{3}$ Independent Research Group Clinical Epidemiology, Helmholtz Zentrum München Deutsches Forschungszentrum für Umwelt und Gesundheit, Neuherberg, Germany
Acknowledgements We thank all members of the Chair of Epidemiology and the BKH Augsburg who are involved in the conduct of the study, especially Martina Wagner, Susanna Sirch, Dorothea Küster and Marion Kötzner. We also thank Dr Ute Amann for her valuable input regarding therapeutic drug monitoring.

Contributors IK and CM conceived the study. IK, CM, MS, JB, JL contributed to design and study protocol. MS, JB, JL contributed to acquisition of data. BM, MS, $\mathrm{JB}$ contributed to patient recruitment, examination/assessment and data collection. IK wrote the manuscript. All authors critically revised and approved the final manuscript.

Funding The authors have not declared a specific grant for this research from any funding agency in the public, commercial or not-for-profit sectors.

Competing interests None declared.

Patient consent for publication Not required.

Ethics approval The study protocol was approved by the Ethics Committee of the Ludwig-Maximilians-Universität München (date of approval: 23 October 2017, reference number: 17-625).

Provenance and peer review Not commissioned; externally peer reviewed.

Open access This is an open access article distributed in accordance with the Creative Commons Attribution Non Commercial (CC BY-NC 4.0) license, which permits others to distribute, remix, adapt, build upon this work non-commercially, and license their derivative works on different terms, provided the original work is properly cited, appropriate credit is given, any changes made indicated, and the use is non-commercial. See: http://creativecommons.org/licenses/by-nc/4.0/.

ORCID iD

Inge Kirchberger http://orcid.org/0000-0003-1967-709X

\section{REFERENCES}

1 World Health Organization. The global burden of disease. 2004 update. Geneva: World Health Organization, 2008.

2 Wittchen $\mathrm{H}-\mathrm{U}$, Jacobi F, Klose M, et al. Depressive Erkrankungen. Gesundheitsbericht-erstattung des Bundes, Heft 51. Berlin: Robert Koch-Institut, 2010.

3 Riihimäki K, Sintonen $\mathrm{H}$, Vuorilehto $\mathrm{M}$, et al. Health-Related quality of life of primary care patients with depressive disorders. Eur Psychiatry 2016;37:28-34.

4 Villoro R, Merino M, Hidalgo-Vega A. Quality of life and use of health care resources among patients with chronic depression. Patient Relat Outcome Meas 2016;7:145-55.

5 Ishak WW, Balayan K, Bresee C, et al. A descriptive analysis of quality of life using patient-reported measures in major depressive disorder in a naturalistic outpatient setting. Qual Life Res 2013;22:585-96.

6 Alonso J, Petukhova M, Vilagut G, et al. Days out of role due to common physical and mental conditions: results from the who world mental health surveys. Mol Psychiatry 2011;16:1234-46.

7 Kamenov K, Caballero FF, Miret M, et al. Which are the most Burdensome functioning areas in depression? A cross-national study. Front Psychol 2016;7:1342.

8 Sobocki P, Jönsson B, Angst J, et al. Cost of depression in Europe. J Ment Health Policy Econ 2006;9:87-98.

9 Wittchen HU, Holsboer F, Jacobi F. Met and unmet needs in the management of depressive disorder in the community and primary care: the size and breadth of the problem. J Clin Psychiatry 2001;62 Suppl 26:23-8.

10 Wittchen HU, Jacobi F, Rehm J, et al. The size and burden of mental disorders and other disorders of the brain in Europe 2010. Eur Neuropsychopharmacol 2011;21:655-79.

11 Melchior H, Schulz H, Härter M, et al. Regionale Unterschiede in Der Diagnostik und Behandlung von Depressionen. Gütersloh: Bertelsmann Stiftung, 2014.

12 Freytag A, Kösters M, Schmauß MKalber J, Günster C, Gerste B, eds. Pharmakotherapie bei depression. Schattauer: Stuttgart, 2014.

13 Kuehner C, Huffziger S. Factors predicting the long-term illness course in a cohort of depressed inpatients. Eur Arch Psychiatry Clin Neurosci 2013;263:413-23.

14 American Psychiatric Association. Practice guideline for the treatment of patients with major depressive disorder. 3rd ED, 2010. https://psychiatryonline.org/ pb/assets/raw/sitewide/practice_ guidelines/guidelines/mdd.pdf

15 DGPPN, BÄK, KBV, AWMF, AkdÄ, BPtK, BApK, DAGSHG, DEGAM, DGPM, DGPS, DGRW (Hrsg.) für die Leitliniengruppe Uniploare 
Depression. S3-Leitlinie/Nationale Versorgungs-leitlinie Unipolare depression - Langfassung, 2. Auflage. version 4, 2015. Available: www.depression.versorgungsleitlinien.de

16 Baumann P, Ulrich S, Eckermann G, et al. The AGNP-TDM expert group consensus guidelines: focus on therapeutic monitoring of antidepressants. Dialogues Clin Neurosci 2005;7:231-47.

17 Hiemke C, Baumann P, Bergemann N, et al. AGNP consensus guidelines for therapeutic drug monitoring in psychiatry: update 2011. Pharmacopsychiatry 2011;44:195-235.

18 Härtel U, Volger E. [Use and acceptance of classical natural and alternative medicine in Germany--findings of a representative population-based survey]. Forsch Komplementarmed Klass Naturheilkd 2004;11:327-34.

19 Bücker B, Groenewold M, Schoefer Y, et al. The use of complementary alternative medicine (cam) in 1001 German adults: results of a population-based telephone survey. Gesundheitswesen 2008;70:e29-36.

20 Linde K, Berner MM, Kriston L. St John's wort for major depression. Cochrane Database Syst Rev 2008;8.

21 Asher GN, Gartlehner G, Gaynes BN, et al. Comparative benefits and harms of complementary and alternative medicine therapies for initial treatment of major depressive disorder: systematic review and metaanalysis. J Altern Complement Med 2017;23:907-19.

22 Gartlehner G, Wagner G, Matyas N, et al. Pharmacological and nonpharmacological treatments for major depressive disorder: review of systematic reviews. BMJ Open 2017;7:e014912.

23 Wagner S, Doering B, Helmreich I, et al. A meta-analysis of executive dysfunctions in unipolar major depressive disorder without psychotic symptoms and their changes during antidepressant treatment. Acta Psychiatr Scand 2012;125:281-92.

24 Jacka FN, Mykletun A, Berk M. Moving towards a population health approach to the primary prevention of common mental disorders. BMC Med 2012;10:149.

25 Rethorst CD, Wipfli BM, Landers DM. The antidepressive effects of exercise: a meta-analysis of randomized trials. Sports Med 2009;39:491-511.

26 Fukumori N, Yamamoto Y, Takegami M, et al. Association between hand-grip strength and depressive symptoms: locomotive syndrome and health outcomes in Aizu cohort study (LOHAS). Age Ageing 2015;44:592-8.

27 Taekema DG, Gussekloo J, Maier AB, et al. Handgrip strength as a predictor of functional, psychological and social health. A prospective population-based study among the oldest old. Age Ageing 2010;39:331-7.

28 van Milligen BA, Lamers F, de Hoop GT, et al. Objective physical functioning in patients with depressive and/or anxiety disorders. $J$ Affect Disord 2011:131:193-9.

29 Knüppel $\mathrm{A}$, Shipley MJ, Llewellyn $\mathrm{CH}$, et al. Sugar intake from sweet food and beverages, common mental disorder and depression: prospective findings from the Whitehall II study. Sci Rep 2017;7:6287.

30 Jacka FN, O'Neil A, Opie R, et al. A randomised controlled trial of dietary improvement for adults with major depression (the 'SMILES' trial). BMC Med 2017;15:23

31 Matins-de-Souza D. Proteomics and metabolomics in psychiatry. Basel, Karger: Advances in Biological Psychiatry, 2014: 29. 92-102.

32 Shao W-hua, Chen J-jun, Fan S-hua, et al. Combined metabolomics and proteomics analysis of major depression in an animal model: perturbed energy metabolism in the chronic mild stressed rat cerebellum. OMICS 2015;19:383-92.

33 Huang $\mathrm{R}$, Wang $\mathrm{K}$, Hu J. Effect of probiotics on depression: a systematic review and meta-analysis of randomized controlled trials. Nutrients 2016;8:483.

34 Wallace CJK, Milev R. The effects of probiotics on depressive symptoms in humans: a systematic review. Ann Gen Psychiatry 2017;16:14

35 Owen L, Corfe B. The role of diet and nutrition on mental health and wellbeing. Proc Nutr Soc 2017;76:425-6.

36 First MB, Spitzer RL, Gibbon M, et al. Structured clinical interview for the DSM-IV axis I disorders (SCID PTSD module. New York: Biometrics Research Department. New York State Psychiatric Institute, 1995.

37 Hamilton M. A rating scale for depression. J Neurol Neurosurg Psychiatry 1960;23:56-62.

38 Hamilton M. The Hamilton Rating Scale for Depression. In: Sartorius N, Ban TA, eds. Assessment of depression. Berlin: Springer, 1986: 143-52.

39 Hautzinger M, Keller F, Kühner CH. BDI-II. Beck-DepressionsInventar. revision. 2. Auflage. Pearson assessment. Frankfurt, 2009
40 Angermeyer MC, Kilian R, Matschinger H. WHOQOL-100 und WHO-QOL-BREF. Handbuch für die deutschsprachige version Der who Instrumente Zur Erfassung von Lebensqualität. Göttingen Bern Toronto, Seattle: Hogrefe, 2000.

41 Brieger P, Röttig S, Marneros A. [Quality of life in unipolar depressive and bipolar affective patients]. Psychiatr Prax 2004;31:304-9.

42 Berlim MT, Pavanello DP, Caldieraro MAK, et al. Reliability and validity of the WHOQOL BREF in a sample of Brazilian outpatients with major depression. Qual Life Res 2005;14:561-4.

43 Rocha NS, Power MJ, Bushnell DM, et al. Cross-Cultural evaluation of the WHOQOL-BREF domains in primary care depressed patients using Rasch analysis. Med Decis Making 2012;32:41-55.

44 Herdman M, Gudex C, Lloyd A, et al. Development and preliminary testing of the new five-level version of EQ-5D (EQ-5D-5L). Qual Life Res 2011;20:1727-36.

45 Huber M, Felix J, Vogelmann M, et al. Health-Related quality of life of the general German population in 2015: results from the EQ-5D-5L. Int J Environ Res Public Health 2017;14:426-E426.

46 Hinz A, Kohlmann T, Stöbel-Richter Y, et al. The quality of life questionnaire EQ-5D-5L: psychometric properties and normative values for the general German population. Qual Life Res 2014;23:443-7.

47 Stark RG, Reitmeir P, Leidl R, et al. Validity, reliability, and responsiveness of the EQ-5D in inflammatory bowel disease in Germany. Inflamm Bowel Dis 2010;16:42-51.

48 Hunger M, Sabariego C, Stollenwerk B, et al. Validity, reliability and responsiveness of the EQ-5D in German stroke patients undergoing rehabilitation. Qual Life Res 2012;21:1205-16.

49 Kolovos S, Bosmans JE, van Dongen JM, et al. Utility scores for different health states related to depression: individual participant data analysis. Qual Life Res 2017;26:1649-58.

50 Bohlscheid-Thomas S, Hoting I, Boeing H. Reproducibility and relative validity of food group intake in a food frequency questionnaire developed for the German part of the EPIC project. European prospective investigation into cancer and nutrition. Int $J$ Epidemiol 1997;26:59S-70.

51 Nagel G, Zoller D, Ruf T, et al. Long-term reproducibility of a foodfrequency questionnaire and dietary changes in the European Prospective Investigation into Cancer and Nutrition (EPIC)Heidelberg cohort. Br J Nutr 2007;98:194-200.

52 Booth M. Assessment of physical activity: an international perspective. Res Q Exerc Sport 2000;71:114-20.

53 Craig CL, Marshall AL, Sjöström M, et al. International physical activity questionnaire: 12 -country reliability and validity. Med Sci Sports Exerc 2003;35:1381-95.

54 McKercher C, Patton GC, Schmidt MD, et al. Physical activity and depression symptom profiles in young men and women with major depression. Psychosom Med 2013;75:366-74.

55 Vancampfort D, Wyckaert S, Sienaert P, et al. Concurrent validity of the International physical activity questionnaire in outpatients with bipolar disorder: comparison with the Sensewear Armband. Psychiatry Res 2016;237:122-6.

56 Ritsher JB, Otilingam PG, Grajales M. Internalized stigma of mental illness: psychometric properties of a new measure. Psychiatry Res 2003;121:31-49.

57 Boyd JE, Adler EP, Otilingam PG, et al. Internalized stigma of mental illness (ISMI) scale: a multinational review. Compr Psychiatry 2014:55:221-31.

58 Sibitz I, Friedrich ME, Unger A, et al. [Internalized Stigma of Schizophrenia: Validation of the German Version of the Internalized Stigma of Mental Illness-Scale (ISMI)]. Psychiatr Prax 2013;40:83-91.

59 Petermann F. Wechsler adult intelligence scale, 2012. Available: https://www.pearsonassessment.de/out/pictures/media/371100.pdf

60 Aschenbrenner S, Tucha O, Lange KW. Regensburger Wortflüssigkeits-Test. Hogrefe: Göttingen Bern Toronto Seattle, 2001.

61 Bäumler G. Farb-Wort-Interferenztest (FWIT) nACh J. R. Stroop. Hogrefe: Göttingen Toronto Zürich, 1985.

62 Kobal G, Hummel T, Sekinger B, et al. "Sniffin' sticks": screening of olfactory performance. Rhinology 1996;34:222-6.

63 Wolfensberger M, Schnieper I, Welge-Lüssen A. Sniffin'Sticks: a new olfactory test battery. Acta Otolaryngol 2000;120:303-6.

64 Deutsche Gesellschaft für Epidemiologie. Leitlinien und Empfehlungen Zur Sicherung von guter epidemiologische praxis. Available: https://www.dgepi.de/fileadmin/pdf/leitlinien/GEP mit Ergaenzung_GPS_Stand_24.02.2009.pdf

65 Nonnemacher M, Nasseh D, Stausberg J. Datenqualität in Der medizinischen Forschung: Leitlinie zum adaptiven management von Datenqualität in Kohortenstudien und Registern; 2014 